\title{
Total Synthesis of (+)-13-Deoxytedanolide
}

\author{
Lisa D. Julian, Jason S. Newcom, and William R. Roush* \\ Department of Chemistry, University of Michigan, Ann Arbor, MI, 48109 \\ e-mail:roush@umich.edu
}

Supporting Information:

Experimental procedures for synthesis of 13-deoxytedanolide from methyl ketone $\mathbf{4}$ and aldehyde 5 
General Experimental Details. All reaction solvents were purified before use. Tetrahydrofuran, dichloromethane, diethyl ether, and toluene were purified by passing through a solvent column composed of activated A-1 alumina. Unless indicated otherwise, all reagents were used as purchased without further purification. All reactions sensitive to moisture or oxygen were conducted under an atmosphere of nitrogen using flame-dried or oven-dried $\left(170{ }^{\circ} \mathrm{C}\right)$ glassware. Crushed $4 \AA$ molecular sieves were activated by thorough flame-drying immediately prior to use.

Proton nuclear magnetic resonance ( ${ }^{1} \mathrm{H}$ NMR) spectra were conducted on a Varian VXR400 spectrometer at $400 \mathrm{MHz}$ or on an Inova-500 spectrometer at $500 \mathrm{MHz}$. Carbon-13 nuclear magnetic resonance $\left({ }^{13} \mathrm{C} \mathrm{NMR}\right)$ spectra were recorded on the aforementioned instruments at 100 $\mathrm{MHz}$ and $125 \mathrm{MHz}$, respectively. The proton signal of residual non-deuterated solvent $(\square 7.26 \mathrm{ppm}$ for $\mathrm{CHCl}_{3}$ ) was used as an internal reference for ${ }^{1} \mathrm{H}$ NMR spectra. For ${ }^{13} \mathrm{C}$ NMR spectra, chemical shifts are reported relative to the $\square 77.2 \mathrm{ppm}$ resonance of $\mathrm{CHCl}_{3}$. Coupling constants are reported in Hz. Infrared (IR) spectra were recorded as films on a Perkin-Elmer Spectrum 1000 FTIR. Optical rotations were measured on a Rudolph Autopol III polarimeter using a quartz cell with 1 $\mathrm{mL}$ capacity and a $10 \mathrm{~cm}$ path length. Mass spectra were recorded on a ZVG 70-250-S spectrometer manufactured by Micromass Corp. (Manchester, UK).

Analytical thin layer chromatography (TLC) was performed on Kieselgel $60 \mathrm{~F}_{254}$ glass plates pre-coated with a $0.25 \mathrm{~mm}$ thickness of silica gel. The TLC plates were visualized with UV light and/or by staining with Hanessian solution (ceric sulfate and ammonium molybdate in aqueous sulfuric acid). Column chromatography was generally performed using Kieselgel 60 (230-400 mesh) silica gel, typically using a 50-100:1 weight ratio of silica gel to crude product.

HPLC purifications were performed by using a HPLC system composed of two Rainin HPXL pumps connected to various Dynamax ${ }^{\circledR}$ axial compression columns packed with $60 \AA$ irregular silica gel. Samples were loaded into the system with a $2 \mathrm{~mL}$ Rheodyne 7125 injector and were detected using either a Rainin Dynamax ${ }^{\circledR}$ UV-C detector or a Dynamax ${ }^{\circledR}$ RI-1 detector.

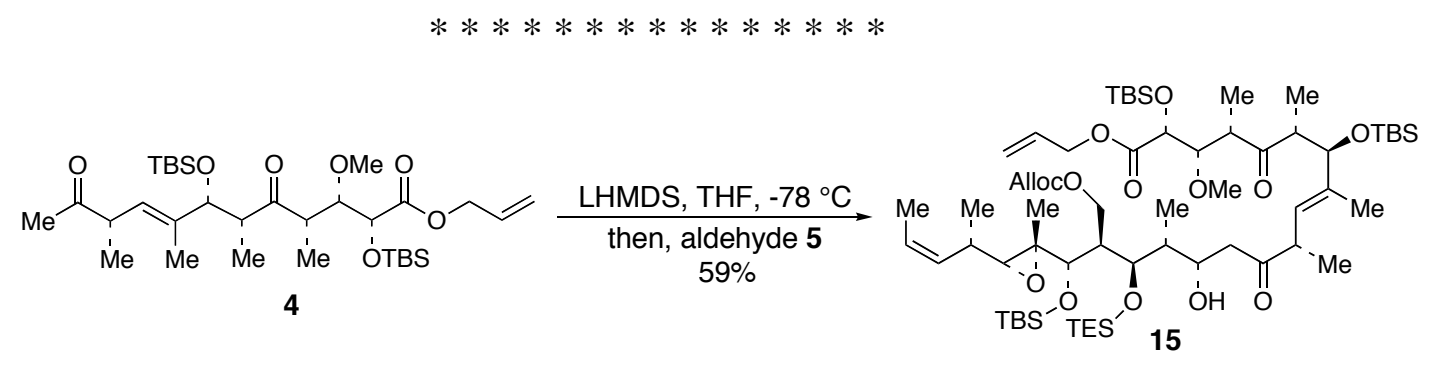

Aldol 15. A stock solution of LHMDS (0.32 M) was prepared by dissolving solid LHMDS (154 mg, $0.92 \mathrm{mmol})$ in THF (2.9 mL). The LHMDS solution (500 $\square \mathrm{L}, 0.16 \mathrm{mmol}$ ) was then added to a flame-dried flask containing $4 \AA$ molecular sieves and cooled to $-78{ }^{\circ} \mathrm{C}$. A solution of ketone 4 
(89 $\mathrm{mg}, 0.15 \mathrm{mmol}$ ) in THF (400 $\square \mathrm{L}$ ) was added dropwise to the LHMDS solution down the side of the flask. The reaction was stirred for $35 \mathrm{~min}$ prior to the addition of aldehyde 5 ( $79 \mathrm{mg}, 0.13$ mmol) in $250 \square \mathrm{L}$ of THF down the side of the flask (with a $100 \square \mathrm{L}$ wash). The mixture was stirrred at $-78{ }^{\circ} \mathrm{C}$ for $20 \mathrm{~min}$, then was poured into a solution of saturated aqueous $\mathrm{NH}_{4} \mathrm{Cl}$ and extracted with $\mathrm{Et}_{2} \mathrm{O}$. The organic layer was washed with brine $(10 \mathrm{~mL})$ and the combined aqueous layers were extracted with $\mathrm{Et}_{2} \mathrm{O}(10 \mathrm{~mL})$. The organic layers were combined, dried over $\mathrm{MgSO}_{4}$, filtered and concentrated in vacuo to give a yellow oil. The crude product was purified by flash column chromatography $(15: 1 \square$ 10:1 hexanes-EtOAc) to afford $11 \mathrm{mg}(14 \%)$ of recovered aldehyde 5, 85 $\mathrm{mg}$ of aldol product and $35 \mathrm{mg}$ of mixed fractions containing recovered ketone and aldol product. The impure material was re-purified under the above conditions to give $24 \mathrm{mg}(27 \%)$ of recovered ketone 4 and an additional $9 \mathrm{mg}$ of pure aldol 15. The total yield of aldol 15 was $94 \mathrm{mg}$ (59\%), a colorless oil: $[\square]_{\mathrm{D}}{ }^{25.6}=+71.4\left(c=0.65 \mathrm{CHCl}_{3}\right) ;{ }^{1} \mathrm{H} \mathrm{NMR}\left(500 \mathrm{MHz}, \mathrm{CDCl}_{3}\right) \square 5.98-5.89(\mathrm{~m}, 2 \mathrm{H})$, 5.48-5.42 (m, 1H), 5.40-5.33 (m, 3H), 5.27 (apparent dq, $J=10.3,1.2 \mathrm{~Hz}, 2 \mathrm{H}$ ), 5.20 (apparent dt, $J$ $=10.4,1.7 \mathrm{~Hz}, 1 \mathrm{H}), 4.68-4.55(\mathrm{~m}, 4 \mathrm{H}), 4.40(\mathrm{br} \mathrm{m}, 1 \mathrm{H}), 4.33(\mathrm{~d}, J=5.9 \mathrm{~Hz}, 1 \mathrm{H}), 4.28(\mathrm{~d}, J=5.6$ $\mathrm{Hz}, 1 \mathrm{H}), 4.18$ (dd, $J=11.2,6.1 \mathrm{~Hz}, 1 \mathrm{H}), 4.09-4.05(\mathrm{~m}, 2 \mathrm{H}), 3.84(\mathrm{dd}, J=5.6,4.2 \mathrm{~Hz}, 1 \mathrm{H}), 3.40$ (s, $3 \mathrm{H}), 3.35$ (apparent ddt, $J=13.7,9.0,6.8 \mathrm{~Hz}, 1 \mathrm{H}), 3.15(\mathrm{~d}, J=9.8 \mathrm{~Hz}, 1 \mathrm{H}), 2.88-2.80(\mathrm{~m}, 3 \mathrm{H}), 2.67$ $\left(\mathrm{A}\right.$ of $\left.\mathrm{ABX}, J_{\mathrm{AB}}=17.3 \mathrm{~Hz}, J_{\mathrm{AX}(\mathrm{obs})}=9.3 \mathrm{~Hz}, 1 \mathrm{H}\right), 2.54(\mathrm{~d}, J=9.0 \mathrm{~Hz}, 1 \mathrm{H}), 2.47-2.41(\mathrm{~m}, 1 \mathrm{H}), 2.36$ $\left(\mathrm{B}\right.$ of $\left.\mathrm{ABX}, J_{\mathrm{AB}}=17.3 \mathrm{~Hz}, J_{\mathrm{BX}(\mathrm{obs})}=3.2 \mathrm{~Hz}, 1 \mathrm{H}\right), 2.16-2.12(\mathrm{~m}, 1 \mathrm{H}), 1.63(\mathrm{~d}, J=1.2 \mathrm{~Hz}, 3 \mathrm{H}), 1.62$ (overlapping dd, $J=6.8,1.7 \mathrm{~Hz}, 3 \mathrm{H}), 1.58-1.55(\mathrm{~m}, 1 \mathrm{H}), 1.3(\mathrm{~s}, 3 \mathrm{H}), 1.14-1.12(\mathrm{~m}, 12 \mathrm{H}), 0.97-0.91$ (m, 30H), 0.88 (s, 9H), 0.71-0.57 (m, 6H), $0.21(\mathrm{~s}, 3 \mathrm{H}), 0.14(\mathrm{~s}, 3 \mathrm{H}), 0.09(\mathrm{~s}, 3 \mathrm{H}), 0.07(\mathrm{~s}, 3 \mathrm{H}), 0.03$ $(\mathrm{s}, 3 \mathrm{H}),-0.03(\mathrm{~s}, 3 \mathrm{H}) ;{ }^{13} \mathrm{C} \mathrm{NMR}\left(125 \mathrm{MHz}, \mathrm{CDCl}_{3}\right) \square 213.2,212.0,171.6,154.9,138.5,132.0$, 131.9, 130.6, 126.4, 119.4, 118.9, 81.3, 79.1, 77.6, 74.9, 74.2, 68.3, 66.3, 66.1, 65.9, 65.3, 63.1, 60.4, 49.2, 46.5, 46.3, 45.9, 45.4, 43.2, 31.6, 26.3, 26.1, 26.0, 19.1, 18.7, 18.6, 18.4, 16.6, 13.5, $13.4,12.7,12.0,11.3,10.1,7.3,5.7,-3.5,-4.2,-4.7,-4.8,-4.9,-5.0$; IR (thin film, $\mathrm{cm}^{-1}$ ) 3539, 2955, 2931, 2881, 2858, 1749, 1711, 1462, 1388, 1362, 1249, 1120, 1076, 993, 904, 838, 778, 726; HRMS (ES+) $m / z$ calcd for $\mathrm{C}_{63} \mathrm{H}_{118} \mathrm{O}_{14} \mathrm{Si}_{4} \mathrm{Na}[\mathrm{M}+\mathrm{Na}]^{+} 1233.7496$, found 1233.7504 .

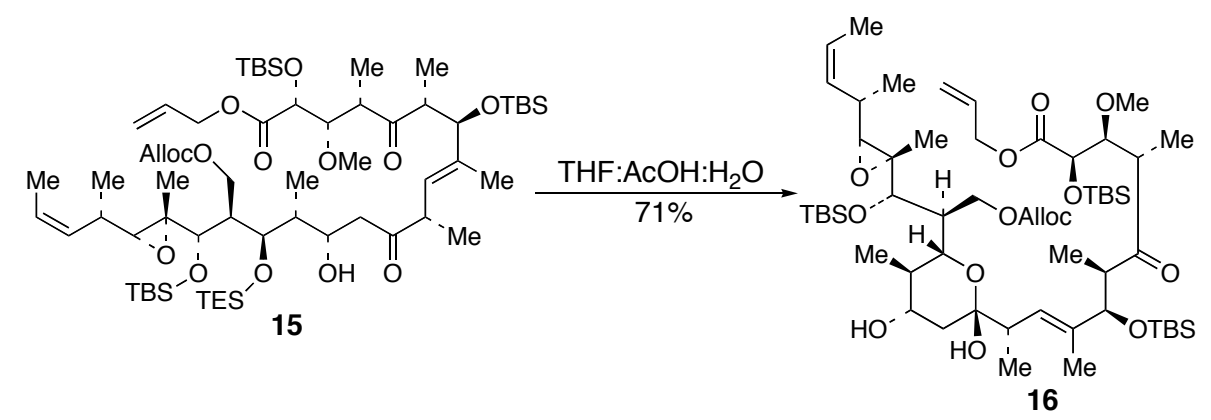

Hemiketal 16. Aldol adduct 15 (50 mg, $0.041 \mathrm{mmol})$ was dissolved in THF (700 $\square \mathrm{L})$, $\mathrm{AcOH}(700 \square \mathrm{L})$ and $\mathrm{H}_{2} \mathrm{O}(200 \square \mathrm{L})$. After being stirred at $23{ }^{\circ} \mathrm{C}$ for $26 \mathrm{~h}$, the reaction mixture was 
quenched by careful addition of saturated aqueous $\mathrm{NaHCO}_{3}$ and diluted with $\mathrm{Et}_{2} \mathrm{O}$. The organic layer was washed with brine $(15 \mathrm{~mL})$ and the combined aqueous layers were extracted with $\mathrm{Et}_{2} \mathrm{O}$ $(15 \mathrm{~mL})$. The organic layers were combined, dried with $\mathrm{MgSO}_{4}$, filtered and concentrated in vacuo to give a yellow oil. Purification of this material via flash column chromatography (10:1 $\square \quad 6: 1$ hexanes-EtOAc) afforded $9.5 \mathrm{mg}$ of recovered 15 (19\%) and $32 \mathrm{mg}$ of product 16 (71\%, 86\% based on recovered starting material) as a colorless oil: $[\square]_{\mathrm{D}}{ }^{25.6}=+31.9\left(c=0.51 \mathrm{CHCl}_{3}\right) ;{ }^{1} \mathrm{H} \mathrm{NMR}(400$ $\left.\mathrm{MHz}, \mathrm{CDCl}_{3}\right) \square 5.92(\mathrm{~m}, 2 \mathrm{H}), 5.47-5.24(\mathrm{~m}, 7 \mathrm{H}), 4.68-4.53(\mathrm{~m}, 4 \mathrm{H}), 4.22(\mathrm{~d}, J=6.6 \mathrm{~Hz}, 1 \mathrm{H}), 4.13-$ $4.09(\mathrm{~m}, 3 \mathrm{H}), 3.95-3.92(\mathrm{~m}, 2 \mathrm{H}), 3.63-3.55(\mathrm{~m}, 1 \mathrm{H}), 3.45(\mathrm{~s}, 3 \mathrm{H}), 3.15(\mathrm{~d}, J=9.5 \mathrm{~Hz}, 1 \mathrm{H}), 3.02-$ 2.95(m, 1H), $2.50(\mathrm{~d}, J=8.8 \mathrm{~Hz}, 1 \mathrm{H}), 2.47-2.34(\mathrm{~m}, 3 \mathrm{H}), 2.15(\mathrm{~d}, J=7.3 \mathrm{~Hz}, 1 \mathrm{H}), 2.08-2.03(\mathrm{~m}$, 2H), $1.88(\mathrm{dd}, J=12.5,4.4 \mathrm{~Hz}, 1 \mathrm{H}), 1.61(\mathrm{dd}, J=6.6,1.1 \mathrm{~Hz}, 3 \mathrm{H}), 1.52(\mathrm{~s}, 3 \mathrm{H}), 1.33(\mathrm{~s}, 3 \mathrm{H}), 1.21$ $(\mathrm{d}, J=7.3 \mathrm{~Hz}, 3 \mathrm{H}), 1.14(\mathrm{~d}, J=7.0 \mathrm{~Hz}, 3 \mathrm{H}), 1.12(\mathrm{~d}, J=6.2 \mathrm{~Hz}, 3 \mathrm{H}), 0.98(\mathrm{~d}, J=6.2 \mathrm{~Hz}, 3 \mathrm{H}), 0.91$ (s, 9H), 0.91 (s, 9H), 0.89 (overlapping d, 3H), 0.87 (s, 9H), 0.18 (s, 3H), 0.08 (s, 3H), 0.07 (s, 3H), 0.07 (s, 3H), 0.05 (s, 3H), -0.03 (s, 3H); ${ }^{13} \mathrm{C} \mathrm{NMR} \mathrm{(100} \mathrm{MHz,} \mathrm{CDCl}_{3}$ ) $\square 213.5,172.2,154.9,136.5$, 131.9, 131.6, 130.6, 129.5, 124.7, 119.7, 118.8, 98.3, 81.8, 80.2, 77.7, 75.8, 71.9, 70.5, 68.3, 66.4, 66.0, 64.7, 63.2, 60.9, 48.2, 48.1, 43.8, 43.0, 42.3, 40.9, 31.6, 26.3, 26.0, 25.9, 19.0, 18.5, 18.4, $18.3,15.4,13.4,13.1,12.7,11.5,11.2,10.3,-3.5,-4.2,-4.8,-4.9,-5.0,-5.5$; IR (thin film, $\mathrm{cm}^{-1}$ ) 3514, 2956, 2931, 2858, 1747, 1710, 1462, 1388, 1362, 1252, 1125, 1077, 993, 903, 838, 778; HRMS (ES+) $m / z$ calcd for $\mathrm{C}_{57} \mathrm{H}_{104} \mathrm{O}_{14} \mathrm{Si}_{3} \mathrm{Na}[\mathrm{M}+\mathrm{Na}]^{+} 1119.6632$, found 1119.6652 .
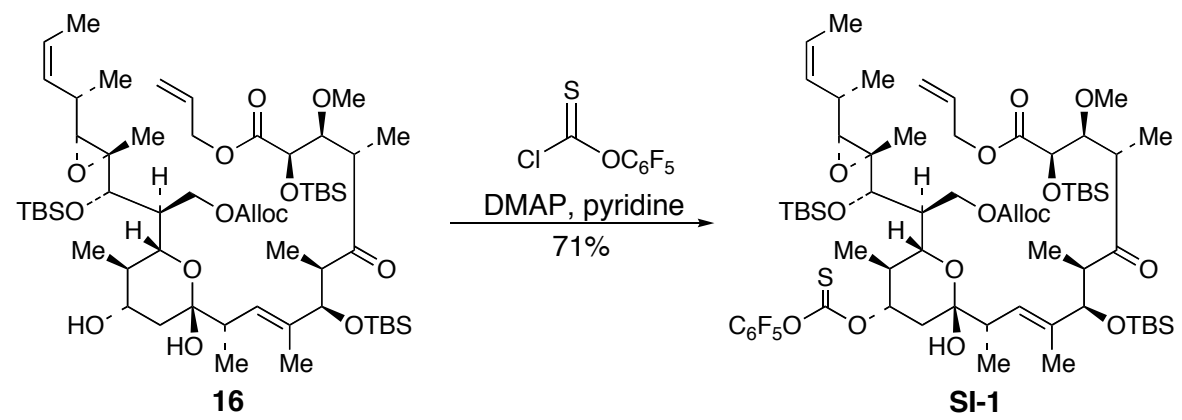

Thiocarbonate SI-1. To a solution of hemiketal 16 (49 mg, $0.045 \mathrm{mmol})$ in $\mathrm{CH}_{2} \mathrm{Cl}_{2}(2.2$ $\mathrm{mL})$ was added pyridine, (1.36 $\mathrm{M}$ in $\mathrm{CH}_{2} \mathrm{Cl}_{2}, 200 \square \mathrm{L}, 0.27 \mathrm{mmol}$ ) pentafluorophenyl chlorothionoformate, $\left(0.68 \mathrm{M}\right.$ in $\left.\mathrm{CH}_{2} \mathrm{Cl}_{2}, 200 \square \mathrm{L}, 0.13 \mathrm{mmol}\right)$ and DMAP $\left(0.08 \mathrm{M}\right.$ in $\mathrm{CH}_{2} \mathrm{Cl}_{2}, 100$ $\square \mathrm{L}, 8 \square \mathrm{mol}$,). After being stirred for $5.5 \mathrm{~h}$ at ambient temperature, the reaction mixture was diluted with saturated aqueous $\mathrm{NH}_{4} \mathrm{Cl}$ and $\mathrm{CH}_{2} \mathrm{Cl}_{2}$. The organic layer was washed with brine (15 mL) and the combined aqueous layers were extracted with $\mathrm{CH}_{2} \mathrm{Cl}_{2}(10 \mathrm{~mL})$. The organic layers were combined, dried over $\mathrm{MgSO}_{4}$, filtered and concentrated in vacuo to give a crude yellow oil. Purification via flash column chromatography (20:1 hexanes-EtOAc) afforded $6 \mathrm{mg}$ of recovered hemiketal 16 (12\%) and $42 \mathrm{mg}$ of the thiocarbonate SI-1 (71\%, 81\% based on recovered 16) as a colorless oil: $[\square]_{\mathrm{D}}{ }^{25.6}=+34.3\left(c=0.35 \mathrm{CHCl}_{3}\right) ;{ }^{1} \mathrm{H} \mathrm{NMR}\left(400 \mathrm{MHz}, \mathrm{CDCl}_{3}\right) \square$ 5.98-5.86 (m, 2H), 
5.48-5.36 (m, 4H), $5.33(\mathrm{dd}, J=5.1,1.1 \mathrm{~Hz}, 1 \mathrm{H}), 5.29-5.23(\mathrm{~m}, 3 \mathrm{H}), 4.67-4.56(\mathrm{~m}, 4 \mathrm{H}), 4.23(\mathrm{~d}, J=$ $5.9 \mathrm{~Hz}, 1 \mathrm{H}), 4.21-4.09(\mathrm{~m}, 4 \mathrm{H}), 3.83(\mathrm{dd}, J=5.9,2.6 \mathrm{~Hz}, 1 \mathrm{H}), 3.36(\mathrm{~s}, 3 \mathrm{H}), 3.18(\mathrm{~d}, J=6.2 \mathrm{~Hz}$, $1 \mathrm{H}), 2.93(\mathrm{app} \mathrm{dq}, J=13.9,7.0 \mathrm{~Hz}, 1 \mathrm{H}), 2.73(\mathrm{dd}, J=7.0,2.6 \mathrm{~Hz}, 1 \mathrm{H}), 2.70(\mathrm{~d}, J=1.8 \mathrm{~Hz}, 1 \mathrm{H})$, 2.53-2.45 (m, 3H), $2.24(\mathrm{dd}, J=12.1,4.8 \mathrm{~Hz}, 1 \mathrm{H}), 2.15-2.04(\mathrm{~m}, 2 \mathrm{H}), 1.62(\mathrm{dd}, J=7.0,1.5 \mathrm{~Hz}$, $3 \mathrm{H}), 1.52(\mathrm{~s}, 3 \mathrm{H}), 1.35(\mathrm{~s}, 3 \mathrm{H}), 1.15-1.10(\mathrm{~m}, 9 \mathrm{H}), 0.99(\mathrm{~d}, J=7.0 \mathrm{~Hz}, 3 \mathrm{H}), 0.97$ (d, J = 7.3 Hz, $3 \mathrm{H}), 0.93$ (s, 9H), 0.89 (s, 9H), 0.88 (s, 9H), 0.20 (s, 3H), 0.16 (s, 3H), 0.07 (s, 3H), 0.05 (s, 3H), 0.04 (s, 3H), -0.02 (s, 3H); ${ }^{13} \mathrm{C}$ NMR (100 MHz, $\left.\mathrm{CDCl}_{3}\right) \square 214.0,191.2,171.6,154.9,138.8,131.8$, 130.5, 127.4, 124.9, 119.3, 119.1, 98.4, 87.4, 80.8, 80.1, 77.7, 75.0, 71.4, 68.4, 66.4, 65.9, 64.7, $63.1,60.4,49.0,46.7,43.9,43.0,38.2,36.9,31.6,26.3,26.0,26.0,19.0,18.5,18.5,18.4,14.6$, $14.4,13.5,12.9,12.1,11.5,10.7,-3.6,-4.2,-4.8,-5.0,-5.3$; IR (thin film, $\mathrm{cm}^{-1}$ ) 2956, 2922, 2857, 1749, 1710, 1523, 1462, 1315, 1253, 1145, 1076, 995, 838, 774; HRMS (ES+) m/z calcd for $\mathrm{C}_{64} \mathrm{H}_{103} \mathrm{O}_{15} \mathrm{~F}_{5} \mathrm{SSi}_{3} \mathrm{Na}[\mathrm{M}+\mathrm{Na}]^{+} 1345.6143$, found 1345.6133 .
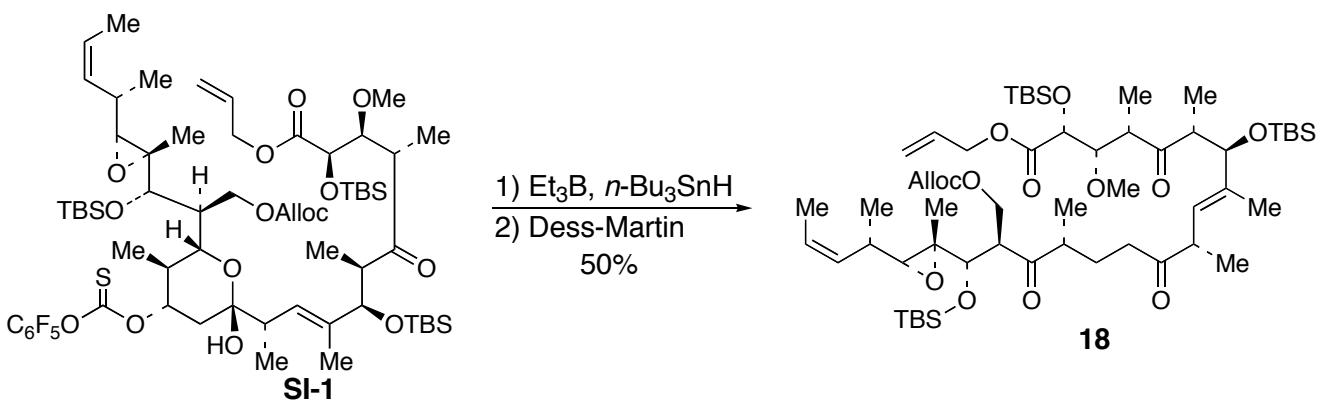

Triketone 18. Thiocarbonate SI-1 (20 mg, $15 \square \mathrm{mol}$ ) was dissolved in $200 \square \mathrm{L}$ of toluene and $n-\mathrm{Bu}_{3} \mathrm{SnH}(0.74 \mathrm{M}$ in toluene, $100 \square \mathrm{L}, 75 \square \mathrm{mol})$ was added. A solution of $\mathrm{Et}_{3} \mathrm{~B}(1 \mathrm{M}$ in hexanes, $200 \square \mathrm{L}$ ) in toluene $(2.4 \mathrm{~mL})$ was prepared in a second flask. A portion of the $\mathrm{Et}_{3} \mathrm{~B}$ solution (100 $\square \mathrm{L}, 7 \square \mathrm{mol}$ ) was then added to the reaction followed by $100 \square \mathrm{L}$ of air. After $15 \mathrm{~min}$, the reaction mixture was concentrated in vacuo and directly purified by flash column chromatography (20:1 hexanes:EtOAc) to provide $10 \mathrm{mg}$ of an unstable hemiketal 17 (62\%). NMR analysis of this material in $\mathrm{CDCl}_{3}$ revealed that it exists $>95 \%$ in the hemiketal form in $\mathrm{CDCl}_{3}$; however, the TLC behavior of the compound suggested that it was an equilibrating mixture of hemiketal and hydroxy ketone. Partial data for the hemiketal 17: $[\square]_{\mathrm{D}}^{25.4}=+27.9\left(c=0.19 \mathrm{CHCl}_{3}\right)$; ${ }^{1} \mathrm{H}$ NMR (400 MHz, $\mathrm{CDCl}_{3}$ ) \5.93-5.82 (m, 2H), 5.43-5.27 (m, 4H), 5.23-5.20 (m, 3H), 4.63-4.49 $(\mathrm{m}, 4 \mathrm{H}), 4.16(\mathrm{~d}, J=5.9 \mathrm{~Hz}, 1 \mathrm{H}), 4.12-4.01(\mathrm{~m}, 3 \mathrm{H}), 3.85(\mathrm{~m}, 1 \mathrm{H}), 3.84$ (overlapping dd, $J=5.9$, $3.3 \mathrm{~Hz}, 1 \mathrm{H}), 3.33$ (s, 3H), $3.12(\mathrm{~d}, J=9.5 \mathrm{~Hz}, 1 \mathrm{H}), 2.96-2.89(\mathrm{~m}, 1 \mathrm{H}), 2.60-2.56(\mathrm{~m}, 1 \mathrm{H}), 2.43-2.40$ (m, 2H), 2.34-2.30 (m, 1H), $2.08(\mathrm{~s}(\mathrm{br}), 1 \mathrm{H}), 2.00-1.98(\mathrm{~m}, 1 \mathrm{H}), 1.59-1.54$ (overlapping $\mathrm{m}, 2 \mathrm{H})$, 1.57 (dd, $J=7.0,1.5 \mathrm{~Hz}, 3 \mathrm{H}), 1.48$ (s, 3H), 1.48-1.44 (overlapping m, 1H), 1.28 (s, 3H), 1.13 (d, $J$ $=7.3 \mathrm{~Hz}, 3 \mathrm{H}), 1.10-1.07(\mathrm{~m}, 6 \mathrm{H}), 0.89(\mathrm{~s}, 9 \mathrm{H}), 0.88$ (overlapping d, 3H), $0.87(\mathrm{~s}, 9 \mathrm{H}), 0.83(\mathrm{~s}, 9 \mathrm{H})$, $0.78(\mathrm{~d}, J=6.2 \mathrm{~Hz}, 3 \mathrm{H}), 0.15$ (s, 3H), 0.09 (s, 3H), 0.03 (s, 3H), 0.02 (s, 3H), 0.00 (s, 3H), -0.06 (s, 
3H); IR (thin film, $\mathrm{cm}^{-1}$ ) 2951, 2926, 2857, 1747, 1710, 1462, 1364, 1251, 1076, 990, 838, 774; HRMS (ES+) $\mathrm{m} / \mathrm{z}$ calcd for $\mathrm{C}_{57} \mathrm{H}_{104} \mathrm{O}_{13} \mathrm{Si}_{3} \mathrm{Na}[\mathrm{M}+\mathrm{Na}]^{+} 1103.6682$, found 1103.6693 .

To a solution of $\mathbf{1 7}(5.5 \mathrm{mg}, 5.1 \square \mathrm{mol})$ in $\mathrm{CH}_{2} \mathrm{Cl}_{2}(3.7 \mathrm{~mL})$ at $0{ }^{\circ} \mathrm{C}$ was added pyridine $(2 \mathrm{M}$ in $\mathrm{CH}_{2} \mathrm{Cl}_{2}, 100 \square \mathrm{L}, 200 \square \mathrm{mol}$ ) and then the Dess-Martin periodinane (15 mg, $35 \square \mathrm{mol}$ ) reagent. The reaction mixture was warmed to $23{ }^{\circ} \mathrm{C}$. After $20 \mathrm{~min}$, another portion of Dess-Martin periodinane ( $6 \mathrm{mg}, 14 \square \mathrm{mmol}$ ) was added and the reaction was allowed to stir for an additional 1.25 h. At this time, saturated aqueous $\mathrm{NaHCO}_{3}$ and solid $\mathrm{Na}_{2} \mathrm{~S}_{2} \mathrm{O}_{3}$ were added to the reaction mixture, and the now heterogeneous mixture was stirred vigorously for $5 \mathrm{~min}$. The organic layer was separated and washed with brine $(5 \mathrm{~mL})$ and the combined aqueous layers were extracted with $\mathrm{CH}_{2} \mathrm{Cl}_{2}(5 \mathrm{~mL})$. The organic layers were combined, dried over $\mathrm{MgSO}_{4}$, filtered and concentrated in vacuo to give a crude yellow oil. Purification of this material via flash column chromatography (15:1 hexanes:EtOAc) afforded $3.9 \mathrm{mg}$ of triketone $18(71 \%)$ as a colorless oil: $\left[\mathrm{C}_{\mathrm{D}}{ }_{\mathrm{D}}^{25.6}=+37.4(c=\right.$ 0.27 $\left.\mathrm{CHCl}_{3}\right) ;{ }^{1} \mathrm{H}$ NMR (400 MHz, $\left.\mathrm{CDCl}_{3}\right) \square 5.98-5.85(\mathrm{~m}, 2 \mathrm{H}), 5.53-5.45(\mathrm{~m}, 1 \mathrm{H}), 5.39-5.31(\mathrm{~m}$, $3 \mathrm{H}), 5.26$ (apparent dd, $J=10.6,1.1 \mathrm{~Hz}, 3 \mathrm{H}), 4.68-4.58(\mathrm{~m}, 4 \mathrm{H}), 4.30-4.26(\mathrm{~m}, 3 \mathrm{H}), 4.00-3.95(\mathrm{~m}$, $1 \mathrm{H}), 3.84(\mathrm{dd}, J=5.5,3.7 \mathrm{~Hz}, 1 \mathrm{H}), 3.43(\mathrm{~d}, J=9.5 \mathrm{~Hz}, 1 \mathrm{H}), 3.38(\mathrm{~s}, 3 \mathrm{H}), 3.37-3.23(\mathrm{~m}, 2 \mathrm{H}), 2.86-$ $2.78(\mathrm{~m}, 2 \mathrm{H}), 2.58-2.53(\mathrm{~m}, 1 \mathrm{H}), 2.53(\mathrm{~d}, J=9.5 \mathrm{~Hz}, 1 \mathrm{H}), 2.46-2.38(\mathrm{~m}, 3 \mathrm{H}), 2.10-1.99(\mathrm{~m}, 1 \mathrm{H})$, $1.62(\mathrm{dd}, J=7.0,1.8 \mathrm{~Hz}, 3 \mathrm{H}), 1.60(\mathrm{~d}, J<1 \mathrm{~Hz}, 3 \mathrm{H}), 1.51-1.41(\mathrm{~m}, 1 \mathrm{H}), 1.33(\mathrm{~s}, 3 \mathrm{H}), 1.13-1.10$ (m, 9H), 1.12 (overlapping d, $J=7.0 \mathrm{~Hz}, 3 \mathrm{H}$ ), 1.07 (d, $J=7.0 \mathrm{~Hz}, 3 \mathrm{H}$ ), 0.90 (s, 9H), 0.86 (s, 9H), 0.84 (s, 9H), 0.12 (s, 3H), 0.08 (s, 3H), 0.06 (s, 3H), 0.02 (s, 3H), -0.04 (s, 3H), -0.06 (s, 3H); ${ }^{13} \mathrm{C}$ NMR $\left(100 \mathrm{MHz}, \mathrm{CDCl}_{3}\right) \square 213.2,211.7,210.9,171.5,154.6,138.1,131.9,131.7,130.6,127.0,125.1$, 119.3, 119.2, 81.1, 77.8, 75.0, 68.8, 66.0, 65.8, 65.6, 62.4, 60.3, 52.5, 49.1, 47.3, 46.2, 46.1, 37.9, 31.6, 26.2, 26.0, 25.9, 25.1, 18.9, 18.4, 18.3, 16.7, 15.0, 13.5, 13.1, 12.9, 11.7, 11.2, -4.2, -4.3, -4.8, -5.0; IR (thin film, $\mathrm{cm}^{-1}$ ) 2951, 2926, 2853, 1749, 1713, 1460, 1388, 1361, 1251, 1074, 993, 836, 777; HRMS (ES+) $m / z$ calcd for $\mathrm{C}_{57} \mathrm{H}_{102} \mathrm{O}_{13} \mathrm{Si}_{3} \mathrm{Na}[\mathrm{M}+\mathrm{Na}]^{+} 1101.6526$, found 1101.6517.

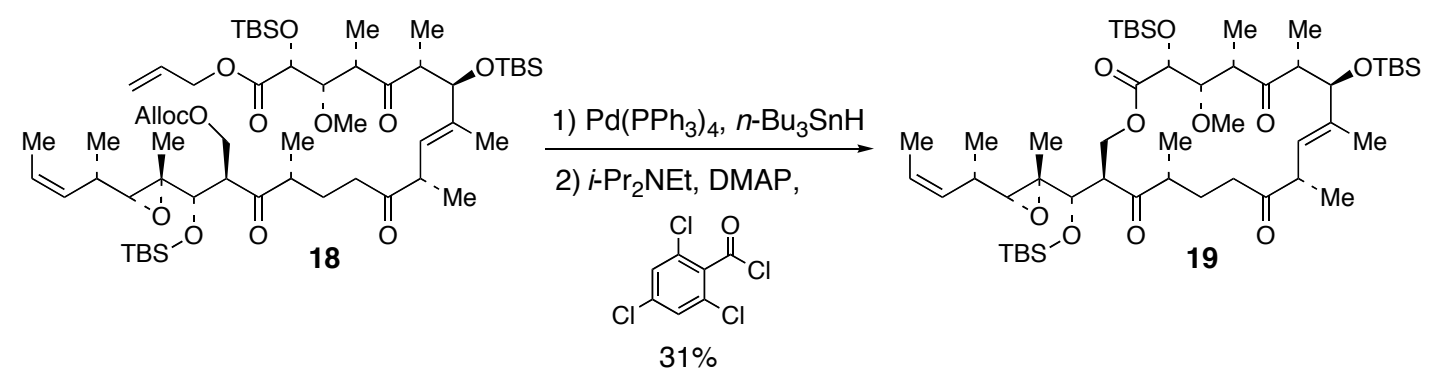

Macrolactone 19. A $0{ }^{\circ} \mathrm{C}$ solution of triketone $18(6 \mathrm{mg}, 5.6 \square \mathrm{mol})$ in $\mathrm{CH}_{2} \mathrm{Cl}_{2}(400 \square \mathrm{L}$ ) was treated with $n-\mathrm{Bu}_{3} \mathrm{SnH}\left(0.3 \mathrm{M}\right.$ in $\left.\mathrm{CH}_{2} \mathrm{Cl}_{2}, 100 \square \mathrm{L}, 30 \square \mathrm{mol}\right)$ and $\mathrm{Pd}\left(\mathrm{PPh}_{3}\right)_{4}\left(1.1 \mathrm{M}^{2} \mathrm{CH}_{2} \mathrm{Cl}_{2}\right.$, $100 \mathrm{~mL}, 0.1$ ( $\mathrm{mol})$. The reaction mixture was stirred for $15 \mathrm{~min}$, then was diluted with saturated aqueous $\mathrm{NaHCO}_{3}$ and $\mathrm{CH}_{2} \mathrm{Cl}_{2}$. The organic layer was washed with saturated aqueous $\mathrm{NH}_{4} \mathrm{Cl}(5$ $\mathrm{mL})$ and brine $(5 \mathrm{~mL})$ and the combined aqueous layers were extracted with $\mathrm{CH}_{2} \mathrm{Cl}_{2}(5 \mathrm{~mL})$. The organic layers were combined, dried over $\mathrm{MgSO}_{4}$, filtered and concentrated in vacuo to give a 
yellow oil. The crude material was filtered through a plug of silica gel (100\% hexanes $\square 100 \%$ EtOAc) to afford the semipure hydroxy acid, which was immediately used in the subsequent macrolactonization reaction.

To a solution of hydroxy acid in $\mathrm{CH}_{2} \mathrm{Cl}_{2}(900 \square \mathrm{L})$ was added $i$ - $\mathrm{Pr}_{2} \mathrm{NEt}\left(0.46 \mathrm{M}\right.$ in $\mathrm{CH}_{2} \mathrm{Cl}_{2}, 50$ $\square \mathrm{L}, 22 \square \mathrm{mol})$, DMAP (0.05 $\mathrm{M}$ in $\left.\mathrm{CH}_{2} \mathrm{Cl}_{2}, 100 \square \mathrm{L}, 5.6 \square \mathrm{mol}\right)$ and trichlorobenzoyl chloride $(0.21 \mathrm{M}$ in $\mathrm{CH}_{2} \mathrm{Cl}_{2}, 50 \square \mathrm{L}, 11 \square \mathrm{mol}$ ). This mixture was stirred for $1 \mathrm{~h}$ at $23{ }^{\circ} \mathrm{C}$, then was diluted with saturated aqueous $\mathrm{NH}_{4} \mathrm{Cl}$ and $\mathrm{CH}_{2} \mathrm{Cl}_{2}$. The organic layer was washed with brine $(5 \mathrm{~mL})$ and the combined aqueous layers were extracted with $\mathrm{CH}_{2} \mathrm{Cl}_{2}(5 \mathrm{~mL})$. The combined organic layers were dried over $\mathrm{MgSO}_{4}$, filtered and concentrated to give a crude yellow oil. Purification of the crude product by flash column chromatography (15:1 hexanes:EtOAc) afforded $1.6 \mathrm{mg}$ of the macrolactone $19(31 \%)$ as a colorless oil: $[\square]_{\mathrm{D}}{ }^{25.6}=+52.5\left(c=0.16 \mathrm{CHCl}_{3}\right) ;{ }^{1} \mathrm{H} \mathrm{NMR}(500 \mathrm{MHz}$, $\left.\mathrm{CDCl}_{3}\right) \square 5.54-5.48(\mathrm{~m}, 1 \mathrm{H}), 5.38-5.33(\mathrm{~m}, 1 \mathrm{H}), 5.06(\mathrm{~d}, J=9.8 \mathrm{~Hz}, 1 \mathrm{H}), 4.29(\mathrm{~d}, J=3.7 \mathrm{~Hz}, 1 \mathrm{H})$, $4.13(\mathrm{dd}, J=10.3,4.1 \mathrm{~Hz}, 1 \mathrm{H}), 4.06(\mathrm{~d}, J=9.5 \mathrm{~Hz}, 1 \mathrm{H}), 4.00-3.95(\mathrm{~m}, 2 \mathrm{H}), 3.41$ (d, $J=10.0 \mathrm{~Hz}$, $1 \mathrm{H}), 3.34(\mathrm{~s}, 3 \mathrm{H}), 3.29-3.24(\mathrm{~m}, 2 \mathrm{H}), 3.11-3.05(\mathrm{~m}, 1 \mathrm{H}), 2.66-2.61(\mathrm{~m}, 2 \mathrm{H}), 2.52(\mathrm{~d}, J=9.0 \mathrm{~Hz}$, $1 \mathrm{H}), 2.47-2.39(\mathrm{~m}, 1 \mathrm{H}), 2.35-2.32(\mathrm{~m}, 2 \mathrm{H}), 2.08-2.00(\mathrm{~m}, 1 \mathrm{H}), 1.62$ (d, $J=0.7 \mathrm{~Hz}, 3 \mathrm{H}), 1.59$ (dd, $J$ $=6.8,1.5 \mathrm{~Hz}, 3 \mathrm{H}), 1.47-1.40(\mathrm{~m}, 1 \mathrm{H}), 1.33(\mathrm{~d}, J=7.3 \mathrm{~Hz}, 3 \mathrm{H}), 1.31(\mathrm{~s}, 3 \mathrm{H}), 1.20(\mathrm{~d}, J=6.8 \mathrm{~Hz}$, $3 \mathrm{H}), 1.16-1.12(\mathrm{~m}, 9 \mathrm{H}), 0.93(\mathrm{~s}, 9 \mathrm{H}), 0.89(\mathrm{~s}, 9 \mathrm{H}), 0.86(\mathrm{~s}, 9 \mathrm{H}), 0.13(\mathrm{~s}, 3 \mathrm{H}), 0.12$ (s, 3H), $0.06(\mathrm{~s}$, $3 \mathrm{H}),-0.02$ (s, 3H), -0.03 (s, 3H); ${ }^{13} \mathrm{C}$ NMR (100 MHz, $\left.\mathrm{CDCl}_{3}\right) \square 214.9,212.9,210.8,170.8,138.0$, 130.6, 128.6, 125.1, 81.0, 79.9, 77.7, 76.2, 65.6, 64.5, 62.3, 60.3, 53.0, 49.7, 49.0, 47.7, 45.9, 37.2, $31.8,26.3,26.2,26.0,25.2,19.0,18.8,18.5,18.4,16.0,15.8,15.2,13.5,11.8,11.6,10.9,-4.2,-4.3$, $-4.5,-4.8,-4.8$; IR (thin film, $\mathrm{cm}^{-1}$ ) 2951, 2926, 2853, 1764, 1718, 1710, 1462, 1364, 1253, 1158, 1076, 988, 902; HRMS (ES+) $m / z$ calcd for $\mathrm{C}_{50} \mathrm{H}_{92} \mathrm{O}_{10} \mathrm{Si}_{3} \mathrm{Na}[\mathrm{M}+\mathrm{Na}]^{+}$959.5896, found 959.5895. In separate experiments, it was found that deprotection of the allyl ester and carbonate proceeded in 74 $\%$ yield after rigorous purification of the hydroxyacid (5:1 hexanes:EtOAc/0.5\% AcOH). Subsequent macrolactonization of the purified seco acid using the conditions described above provided macrolactone $\mathbf{1 9}$ in $40 \%$ yield.

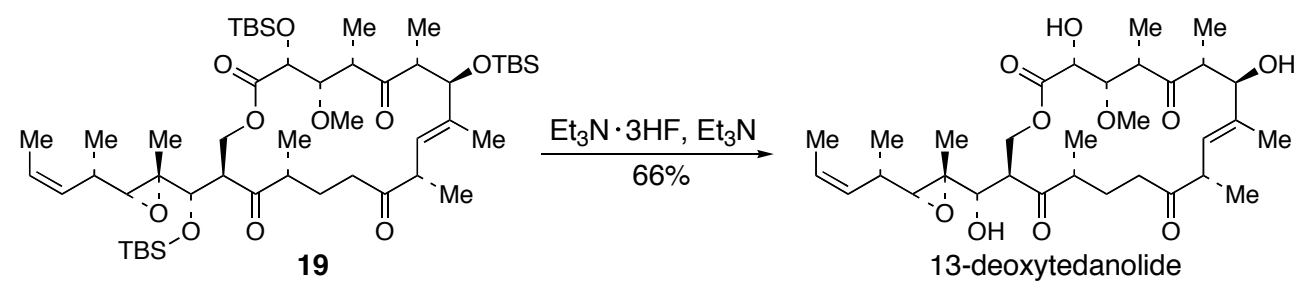

(+)-13-Deoxytedanolide (2). To a solution of macrolactone 19 (1.2 mg, $1.3 \square \mathrm{mol})$ in $\mathrm{CH}_{3} \mathrm{CN}\left(200 \square \mathrm{L}\right.$ ) was added $\mathrm{Et}_{3} \mathrm{~N} \cdot 3 \mathrm{HF}\left(150 \square \mathrm{L}\right.$ ) followed by $\mathrm{Et}_{3} \mathrm{~N}$ (130 $\square \mathrm{L}$ ). This mixture was allowed to stand at ambient temperature for $4 \mathrm{~d}$ (without stirring), then was slowly added to saturated aqueous $\mathrm{NaHCO}_{3}$. The resulting mixture was diluted with $\mathrm{Et}_{2} \mathrm{O}$ and stirred for $10 \mathrm{~min}$. The organic layer was washed with saturated aqueous $\mathrm{NaHCO}_{3}(2 \square 5 \mathrm{~mL})$ and brine $(5 \mathrm{~mL})$ and the 
combined aqueous layers were extracted with $\mathrm{Et}_{2} \mathrm{O}(2 \square 5 \mathrm{~mL})$. The organic layers were combined, dried over $\mathrm{MgSO}_{4}$, filtered and concentrated in vacuo to give an oil. Purification of this material via flash column chromatography (1:1 hexanes:EtOAc) afforded $0.5 \mathrm{mg}(66 \%)$ of 13-deoxytedanolide (2) as a white solid: $[\square]_{\mathrm{D}}{ }^{24.3}=+85.6\left(c=0.09 \mathrm{CHCl}_{3}\right)\left(\right.$ lit. value $\left.{ }^{1}=+84.4, c=0.26 \mathrm{CHCl}_{3}\right) ;{ }^{1} \mathrm{H} \mathrm{NMR}$ $\left(500 \mathrm{MHz}, \mathrm{CD}_{3} \mathrm{OD}\right) \square 5.49(\mathrm{dq}, J=11.0,6.8 \mathrm{~Hz}, 1 \mathrm{H}), 5.33(\mathrm{ddq}, J=10.7,10.3,1.7 \mathrm{~Hz}, 1 \mathrm{H}), 5.29$ $(\mathrm{dq}, J=9.5,1.2, \mathrm{~Hz}, 1 \mathrm{H}), 4.21(\mathrm{dd}, J=10.5,4.4 \mathrm{~Hz}, 1 \mathrm{H}), 4.02(\mathrm{dd}, J=12.0,10.7 \mathrm{~Hz}, 1 \mathrm{H}), 3.97$ (d, $J=10.3 \mathrm{~Hz}, 1 \mathrm{H}), 3.68(\mathrm{~d}, J=1.7 \mathrm{~Hz}, 1 \mathrm{H}), 3.61(\mathrm{dd}, J=9.5,1.7 \mathrm{~Hz}, 1 \mathrm{H}), 3.41-3.35$ (overlapping $\mathrm{m}$, $2 \mathrm{H}), 3.35(\mathrm{~s}, 3 \mathrm{H}), 3.17(\mathrm{~d}, J=10.0 \mathrm{~Hz}, 1 \mathrm{H}), 3.15-3.09(\mathrm{~m}, 2 \mathrm{H}), 2.81-2.78(\mathrm{~m}, 1 \mathrm{H}), 2.61(\mathrm{~d}, J=9.0$ $\mathrm{Hz}, 1 \mathrm{H}), 2.51-2.48(\mathrm{~m}, 1 \mathrm{H}), 2.33-2.26(\mathrm{~m}, 2 \mathrm{H}), 2.00-1.93(\mathrm{~m}, 1 \mathrm{H}), 1.64(\mathrm{~d}, J=1.2 \mathrm{~Hz}, 3 \mathrm{H}), 1.63$ (dd, $J=6.8,1.7 \mathrm{~Hz}, 3 \mathrm{H}), 1.58$ (overlapping m, 1H), 1.36 (s, 3H), 1.28 (d, J=7.1 Hz, 3H), 1.24 (d, $J$ $=7.1 \mathrm{~Hz}, 3 \mathrm{H}), 1.13(\mathrm{~d}, J=7.3 \mathrm{~Hz}, 3 \mathrm{H}), 1.11(\mathrm{~d}, J=6.8 \mathrm{~Hz}, 3 \mathrm{H}), 1.03(\mathrm{~d}, J=6.8 \mathrm{~Hz}, 3 \mathrm{H}) ;{ }^{13} \mathrm{C} \mathrm{NMR}$ (125 MHz, $\left.\mathrm{CD}_{3} \mathrm{OD}\right) \square 217.3,215.6,213.8,173.5,138.3,131.8,130.1,126.0,85.1,80.3,77.6,72.5$, 67.5, 64.1, 61.2, 53.2, 51.1, (49.3, 49.1 overlapping $\left.\mathrm{w} / \mathrm{CD}_{3} \mathrm{OD}\right), 46.2$, 38.7, 32.4, 26.0, 18.7, 16.2, 15.6, 15.1, 13.5, 11.4, 10.4; IR (thin film, $\mathrm{cm}^{-1}$ ) 3439, 2966, 2853, 1750, 1710, 1455, 1374, 1268, 1091, 1032, 981, 755; HRMS (ES+) $m / z$ calcd for $\mathrm{C}_{32} \mathrm{H}_{50} \mathrm{O}_{10} \mathrm{Na}[\mathrm{M}+\mathrm{Na}]^{+}$617.3302, found 617.3295.

The spectroscopic properties of synthetic (+)-13-deoxytedanolide compared very favorably with data for natural 13-deoxytedanolide published by Fusetani, ${ }^{1}$ and for synthetic 13deoxytedanolide published by Smith. ${ }^{2,3}$ Tables containing comparative ${ }^{1} \mathrm{H}$ and ${ }^{13} \mathrm{C}$ NMR data appear on the following pages.

\section{Literature Cited in the Supporting Information}

(1) Fusetani, N.; Sugawara, T.; Matsunaga, S.; Hirota, H. J. Org. Chem 1991, 56, 4971.

(2) Smith, A. B.; Adams, C. M.; Lodise Barbosa, S. A.; Degnan, A. P. J. Am. Chem. Soc. 2003, 125,350 .

(3) Smith, A. B.; Adams, C. M.; Lodise Barbosa, S. A.; Degnan, A. P. Proc. Natl. Acad. Sci. USA 2004, 101, 12042. 
壳志志志志志志志

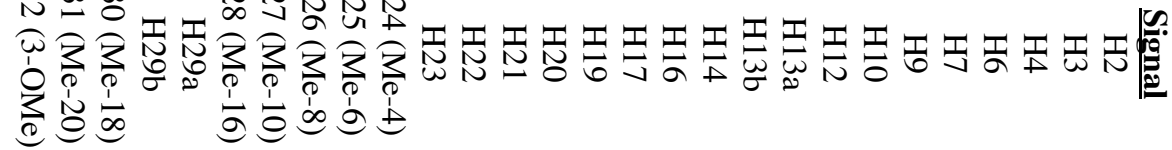

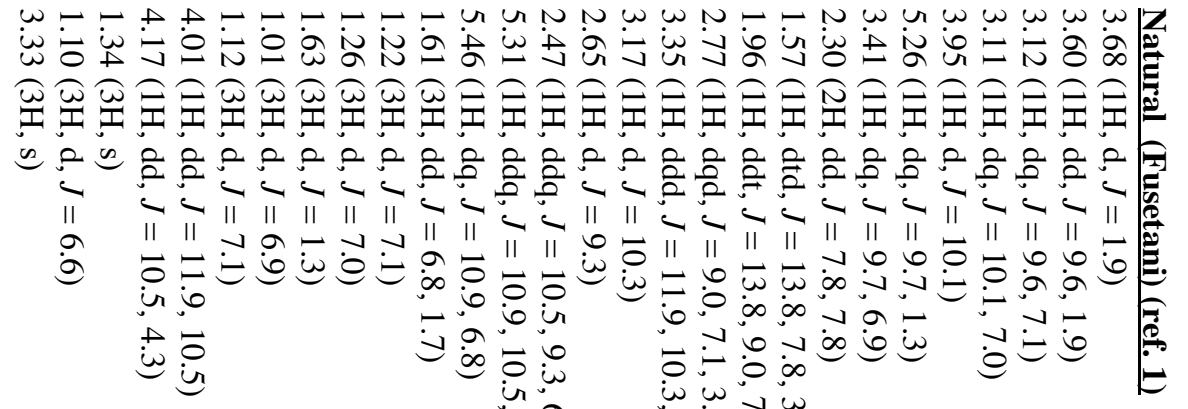

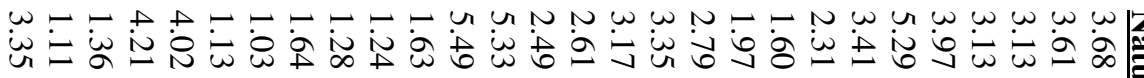

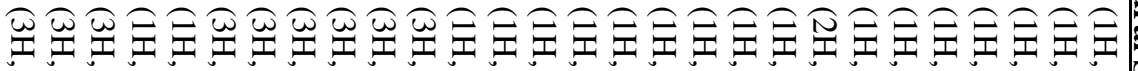

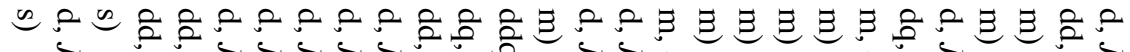

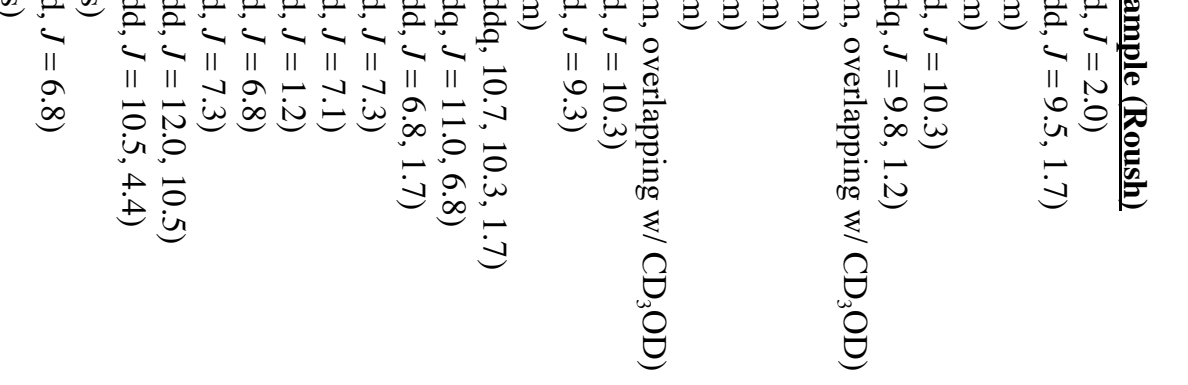

-

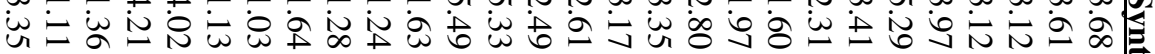

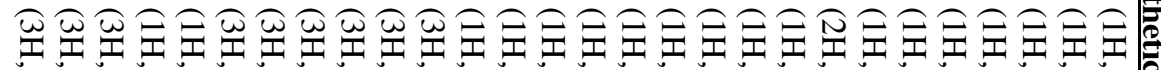

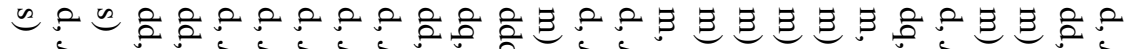

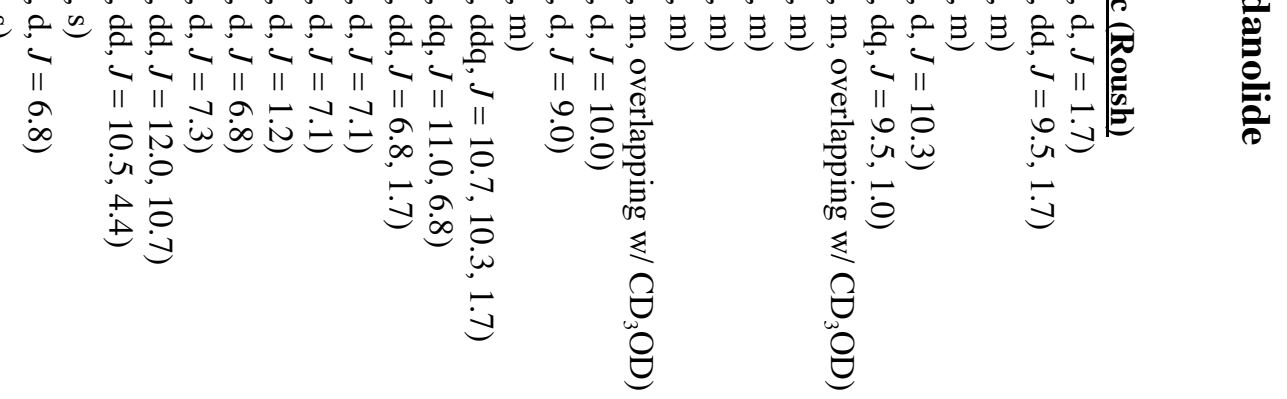

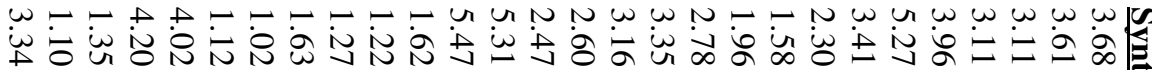

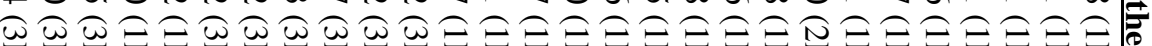

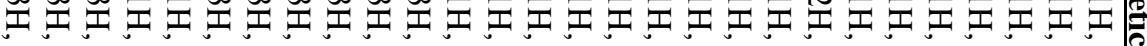

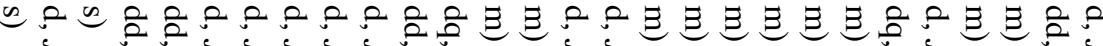

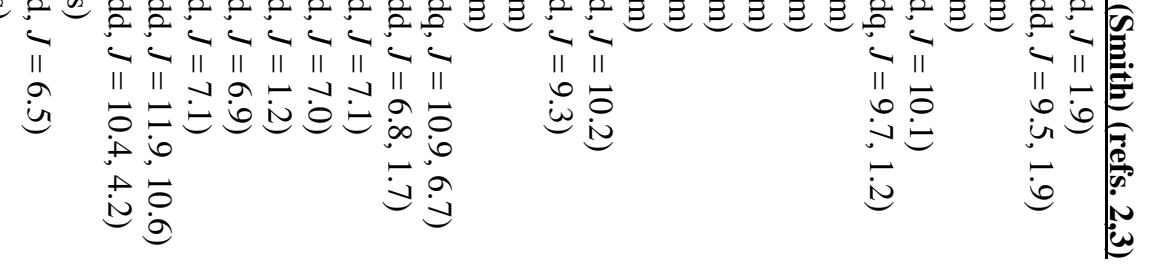




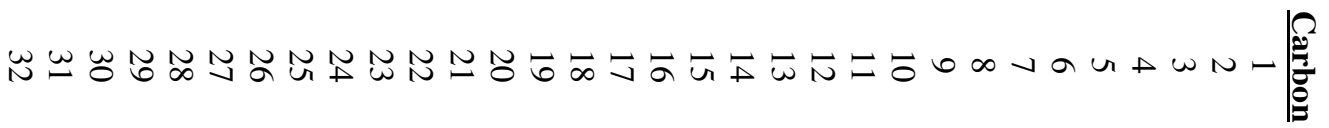

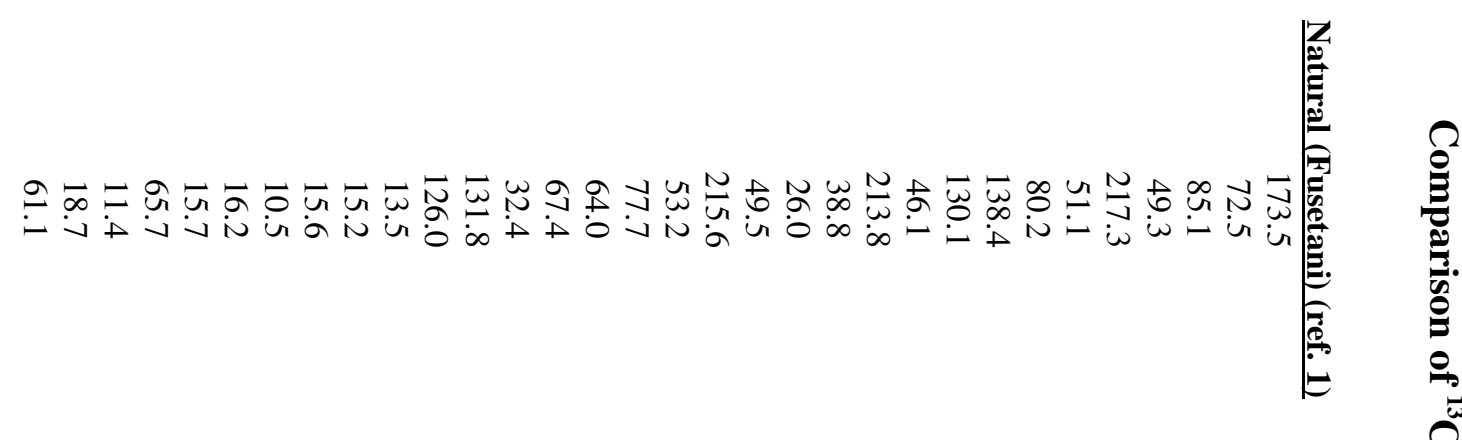

$\frac{n}{0}$

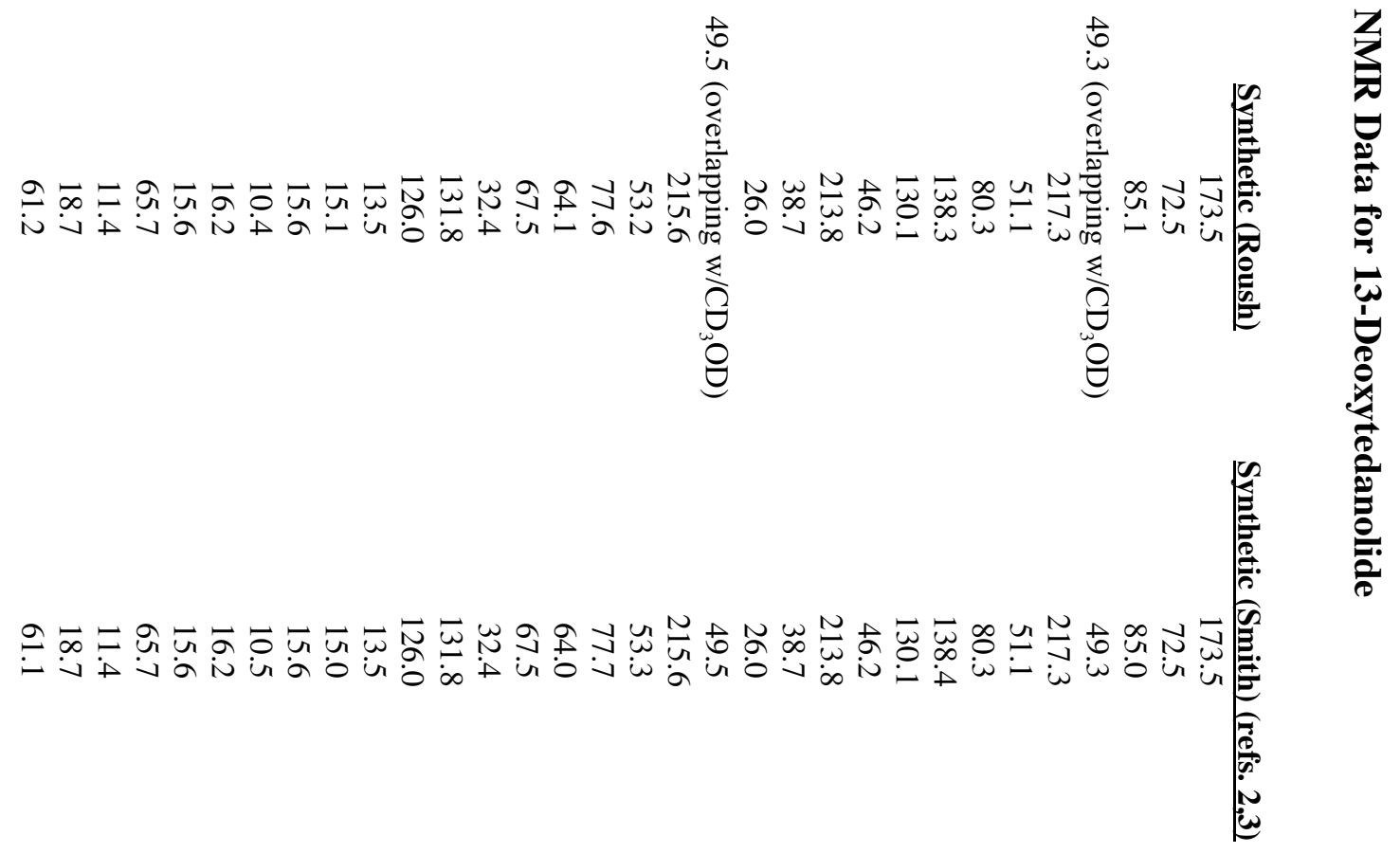

